\title{
Trimaximal mixing with a texture zero
}

\author{
Radha Raman Gautam* \\ Department of Physics, Himachal Pradesh University, Shimla 171005, India
}

(Received 17 December 2017; published 15 March 2018)

\begin{abstract}
We analyze neutrino mass matrices having one texture zero, assuming that the neutrino mixing matrix has either its first $\left(\mathrm{TM}_{1}\right)$ or second $\left(\mathrm{TM}_{2}\right)$ column identical to that of the tribimaximal mixing matrix. We found that all six possible one texture-zero neutrino mass matrices are compatible with the present neutrino oscillation data when combined with $\mathrm{TM}_{1}$ or $\mathrm{TM}_{2}$ mixing. These textures have interesting predictions for the presently unknown parameters such as the effective Majorana neutrino mass, the Dirac $C P$ violating phase, and the neutrino mass scale. We also present a way to theoretically realize some of these textures using $A_{4}$ symmetry within the framework of the type-I + II seesaw mechanism.
\end{abstract}

DOI: 10.1103/PhysRevD.97.055022

\section{INTRODUCTION}

Various neutrino oscillation experiments in the last decade or so have measured the three lepton mixing angles, and it is now clear that the flavor mixing in the lepton sector is quite large as compared to the quark sector. Non-Abelian discrete flavor symmetries have been extensively utilized to explain the large mixing in the lepton sector [1]. Among the most widely studied lepton mixing patterns obtained using discrete non-Abelian symmetries is the tribimaximal (TBM) mixing pattern [2],

$$
U_{\mathrm{TBM}}=\left(\begin{array}{ccc}
-\frac{\sqrt{2}}{\sqrt{3}} & \frac{1}{\sqrt{3}} & 0 \\
\frac{1}{\sqrt{6}} & \frac{1}{\sqrt{3}} & -\frac{1}{\sqrt{2}} \\
\frac{1}{\sqrt{6}} & \frac{1}{\sqrt{3}} & \frac{1}{\sqrt{2}}
\end{array}\right),
$$

which predicts the reactor mixing angle $\theta_{13}$ to be zero, a maximal atmospheric mixing angle, i.e., $\theta_{23}=\frac{\pi}{4}$, and the solar mixing angle $\theta_{12}=\sin ^{-1}(1 / \sqrt{3})$. However, recent neutrino oscillation experiments have found $\theta_{13}$ to be nonzero [3], necessitating some modifications to the TBM mixing scheme to make it compatible with the present experimental data. In this context, it has been observed that the first or the second column of TBM mixing matrix can still accommodate the recent neutrino oscillation data [4]. When the second column of TBM mixing matrix remains intact while the other columns

\footnotetext{
*gautamrrg@gmail.com
}

Published by the American Physical Society under the terms of the Creative Commons Attribution 4.0 International license. Further distribution of this work must maintain attribution to the author(s) and the published article's title, journal citation, and DOI. Funded by SCOAP ${ }^{3}$. deviate from TBM values, we denote that mixing matrix as $\mathrm{TM}_{2}$ [5]. Similarly, if the first column of TBM remains intact and other columns deviate from TBM, we denote it by $\mathrm{TM}_{1}$ [5]. When either of these columns of TBM remain intact in the lepton mixing matrix, we can parametrize the mixing matrix in terms of one mixing angle and one $C P$ violating Dirac phase along with two Majorana phases $[4,5]$.

There are many other approaches which have been used to explain neutrino mixing; some of these are texture zeros [6], vanishing cofactors [7], hybrid textures [8], etc. Out of these, texture zeros have been quite successful in explaining mixing in both the quark and the lepton sectors. In the basis where the charged lepton mass matrix is diagonal there are seven patterns of two texture zeros which are compatible with the current neutrino oscillation data [6]. There are six possible patterns of one texture zero in the neutrino mass matrix which are shown in Table I. All of these are compatible with the present experimental data [9].

Neutrino mass matrices with two texture zeros in combination with $\mathrm{TM}_{2}$ mixing have been studied in Ref. [10], where it has been found that only two patterns, namely $A_{1}$ and $A_{2}$ (with texture zeros at $(1,1),(1,2)$, and $(1,1),(1,3)$ entries, respectively), can satisfy the present

TABLE I. Six allowed patterns of one texture zero in the neutrino mass matrix.

\begin{tabular}{lc}
\hline \hline Pattern & Constraining equation \\
\hline I & $M_{e e}=0$ \\
II & $M_{e \mu}=0$ \\
III & $M_{e \tau}=0$ \\
IV & $M_{\mu \mu}=0$ \\
V & $M_{\mu \tau}=0$ \\
VI & $M_{\tau \tau}=0$ \\
\hline \hline
\end{tabular}


experimental data when we combine two texture zeros with $\mathrm{TM}_{2}$ mixing. The combination of $\mathrm{TM}_{1}$ mixing with two texture zeros has been studied in Ref. [11] and, similar to the $\mathrm{TM}_{2}$ case, it has been found that only patterns $A_{1}$ and $A_{2}$ of two texture zeros are compatible with the present neutrino oscillation data when combined with $\mathrm{TM}_{1}$ mixing. This approach of combining texture zeros with $\mathrm{TM}_{1} / \mathrm{TM}_{2}$ mixing turns out to be very fruitful as it leads to very predictive texture structures of the neutrino mass matrix. In the present work, we study neutrino mass matrices having one texture zero in combination with $\mathrm{TM}_{2} / \mathrm{TM}_{1}$ mixing.

\section{TM 2 MIXING AND ONE TEXTURE ZERO}

\section{A. $\mathbf{T M}_{2}$ mixing}

A neutrino mass matrix with TBM mixing can be diagonalized as

$$
M_{\text {diag }}=U_{\mathrm{TBM}}^{T} M_{\mathrm{TBM}} U_{\mathrm{TBM}},
$$

where $U_{\mathrm{TBM}}$ contains the eigenvectors $t=\left(-\frac{\sqrt{2}}{\sqrt{3}} \frac{1}{\sqrt{6}} \frac{1}{\sqrt{6}}\right)^{T}$, $u=\left(\frac{1}{\sqrt{3}} \frac{1}{\sqrt{3}} \frac{1}{\sqrt{3}}\right)^{T}$, and $v=\left(0 \frac{-1}{\sqrt{2}} \frac{1}{\sqrt{2}}\right)^{T}$. The diagonal matrix $M_{\text {diag }}$ is given as

$$
M_{\text {diag }}=\left(\begin{array}{ccc}
m_{1} & 0 & 0 \\
0 & e^{2 i \alpha} m_{2} & 0 \\
0 & 0 & e^{2 i \beta} m_{3}
\end{array}\right),
$$

where $m_{1}, m_{2}$, and $m_{3}$ are the three neutrino masses and $\alpha$ and $\beta$ are the two Majorana phases. The mass matrix $M_{\mathrm{TBM}}$ is invariant under the transformations $G_{t}, G_{u}$, and $G_{v}$; i.e., $G_{t}^{T} M_{\mathrm{TBM}} G_{t}=M_{\mathrm{TBM}}, G_{u}^{T} M_{\mathrm{TBM}} G_{u}=M_{\mathrm{TBM}}$, and $G_{v}^{T} M_{\mathrm{TBM}} G_{v}=M_{\mathrm{TBM}}$ with $G_{t}=1-2 t t^{T}, G_{u}=1-2 u u^{T}$, and $G_{v}=1-2 v v^{T}$. The transformation matrices $G_{u}$ and $G_{v}$ correspond to the magic symmetry [12] and the $\mu-\tau$ symmetry [12], respectively. $G_{i}(i=t, u, v)$ are generators of the $Z_{2}$ group. A $3 \times 3$ neutrino mass matrix with TBM symmetry is invariant under the $Z_{2}^{t} \times Z_{2}^{u} \times Z_{2}^{v}$ group. Recently, neutrino oscillation experiments have confirmed a nonzero $\theta_{13}$; thus, the neutrino mass matrix $M_{\nu}$ cannot remain invariant under the $\mu-\tau$ symmetry transformation $G_{v}$. However, the neutrino mass matrix can still be invariant under the magic symmetry transformation $G_{u}$ as the experimental data are still compatible with the magic symmetry. The mixing matrix which corresponds to the magic symmetry is known as the trimaximal mixing $\left(\mathrm{TM}_{2}\right)$ matrix and can be parametrized as $[4,5,12-14]$

$$
U_{\mathrm{TM}_{2}}=\left(\begin{array}{ccc}
\sqrt{\frac{2}{3}} \cos \theta & \frac{1}{\sqrt{3}} & \sqrt{\frac{2}{3}} \sin \theta \\
-\frac{\cos \theta}{\sqrt{6}}+\frac{e^{-i \phi} \sin \theta}{\sqrt{2}} & \frac{1}{\sqrt{3}} & -\frac{\sin \theta}{\sqrt{6}}-\frac{e^{-i \phi} \cos \theta}{\sqrt{2}} \\
-\frac{\cos \theta}{\sqrt{6}}-\frac{e^{-i \phi} \sin \theta}{\sqrt{2}} & \frac{1}{\sqrt{3}} & -\frac{\sin \theta}{\sqrt{6}}+\frac{e^{-i \phi} \cos \theta}{\sqrt{2}}
\end{array}\right) .
$$

The $\mathrm{TM}_{2}$ mixing matrix has its middle column fixed to the TBM value $(u)$, which leaves only two free parameters $(\theta$ and $\phi)$ in $U_{\mathrm{TM}_{2}}$ after we take into account the unitarity constraints. The neutrino mass matrix corresponding to $\mathrm{TM}_{2}$ mixing is given as

$$
M_{\mathrm{TM}_{2}}=U_{\mathrm{TM}_{2}}^{*} M_{\mathrm{diag}} U_{\mathrm{TM}_{2}}^{\dagger} .
$$

\section{B. One zero in $\boldsymbol{M}_{\mathrm{TM}_{2}}$}

A neutrino mass matrix with $\mathrm{TM}_{2}$ mixing can be parametrized as [15]

$$
M_{\mathrm{TM}_{2}}=\left(\begin{array}{ccc}
a & b & c \\
b & d & a+c-d \\
c & a+c-d & b-c+d
\end{array}\right) .
$$

The constraint equations for all the patterns with one texture zero and $\mathrm{TM}_{2}$ mixing can be obtained by substituting the respective texture zero constraints from Table I into Eq. (6). In the diagonal charged lepton mass matrix basis, all the six patterns of one texture zero in the neutrino mass matrix are compatible with the present experimental data. The combination of these one texture zero patterns with $\mathrm{TM}_{2}$ mixing is bound to produce very predictive forms of neutrino mass matrices.

The neutrino mass matrices with one texture zero and $\mathrm{TM}_{2}$ mixing are given below:

$$
\begin{aligned}
M_{\mathrm{TM}_{2}}^{\mathrm{I}} & =\left(\begin{array}{ccc}
0 & b & c \\
b & d & c-d \\
c & c-d & b-c+d
\end{array}\right) \\
M_{\mathrm{TM}_{2}}^{\mathrm{II}}= & \left(\begin{array}{ccc}
a & 0 & c \\
0 & d & a+c-d \\
c & a+c-d & -c+d
\end{array}\right) \\
M_{\mathrm{TM}_{2}}^{\mathrm{II}}= & \left(\begin{array}{ccc}
a & b & 0 \\
b & d & a-d \\
0 & a-d & b+d
\end{array}\right) \\
M_{\mathrm{TM}_{2}}^{\mathrm{IV}} & =\left(\begin{array}{ccc}
a & b & c \\
b & 0 & a+c \\
c & a+c & b-c
\end{array}\right) \\
M_{\mathrm{TM}_{2}}^{\mathrm{V}} & =\left(\begin{array}{ccc}
a & b & c \\
b & a+c & 0 \\
c & 0 & a+b
\end{array}\right) \\
M_{\mathrm{TM}_{2}}^{\mathrm{VI}} & =\left(\begin{array}{ccc}
a & b & c \\
b & c-b & a+b \\
c & a+b & 0
\end{array}\right) .
\end{aligned}
$$

The above mass matrices [Eqs. (7) to (12)] can be rewritten as 
$M_{\mathrm{TM}_{2}}^{\mathrm{I}}=\left(\begin{array}{ccc}0 & b & c \\ b & c-\Delta & \Delta \\ c & \Delta & b-\Delta\end{array}\right)$ where $\Delta=c-d$

$M_{\mathrm{TM}_{2}}^{\mathrm{II}}=\left(\begin{array}{ccc}a & 0 & c \\ 0 & c-\Delta & a+\Delta \\ c & a+\Delta & -\Delta\end{array}\right) \quad$ where $\Delta=c-d$

$M_{\mathrm{TM}_{2}}^{\mathrm{III}}=\left(\begin{array}{ccc}a & b & 0 \\ b & d & a-d \\ 0 & a-d & b+d\end{array}\right)$

$M_{\mathrm{TM}_{2}}^{\mathrm{IV}}=\left(\begin{array}{ccc}\Lambda-c & b & c \\ b & 0 & \Lambda \\ c & \Lambda & b-c\end{array}\right) \quad$ where $\Lambda=a+c$

$M_{\mathrm{TM}_{2}}^{\mathrm{V}}=\left(\begin{array}{ccc}a & \Omega-a & c \\ \Omega-a & a+c & 0 \\ c & 0 & \Omega\end{array}\right)$ where $\Omega=a+b$

$M_{\mathrm{TM}_{2}}^{\mathrm{VI}}=\left(\begin{array}{ccc}\Omega-b & b & c \\ b & c-b & \Omega \\ c & \Omega & 0\end{array}\right) \quad$ where $\Omega=a+b$

All the six patterns from Eqs. (13) to (18) can be written as a linear combination of following matrices

$\begin{aligned} p_{12}=\left(\begin{array}{lll}0 & 1 & 0 \\ 1 & 0 & 0 \\ 0 & 0 & 1\end{array}\right), & p_{13}=\left(\begin{array}{lll}0 & 0 & 1 \\ 0 & 1 & 0 \\ 1 & 0 & 0\end{array}\right), \\ p_{23}=\left(\begin{array}{lll}1 & 0 & 0 \\ 0 & 0 & 1 \\ 0 & 1 & 0\end{array}\right), & b_{12}=\left(\begin{array}{ccc}-1 & 1 & 0 \\ 1 & -1 & 0 \\ 0 & 0 & 0\end{array}\right), \\ b_{13}=\left(\begin{array}{ccc}-1 & 0 & 1 \\ 0 & 0 & 0 \\ 1 & 0 & -1\end{array}\right), & b_{23}=\left(\begin{array}{ccc}0 & 0 & 0 \\ 0 & -1 & 1 \\ 0 & 1 & -1\end{array}\right),\end{aligned}$

where the first three are the symmetric permutation matrices and the last three are in block diagonal form, e.g. $M_{\mathrm{TM}_{2}}^{\mathrm{I}}$ is obtained as a linear combination of $p_{12}, p_{13}$, and $b_{23}$ :

$$
M_{\mathrm{TM}_{2}}^{\mathrm{I}}=\Delta b_{23}+b p_{12}+c p_{13} .
$$

Similarly, we can construct other patterns. This representation brings all the patterns on equal footing; i.e., all the one texture zero patterns with $\mathrm{TM}_{2}$ mixing are made up of simple combinations of two symmetric permutation matrices and a block diagonal matrix. The above decomposition into permutation and block diagonal matrices also helps in the symmetry realization of these patterns.

A neutrino mass matrix with $\mathrm{TM}_{2}$ mixing is diagonalized by the mixing matrix $U=U_{\mathrm{TM}_{2}}$ given in Eq. (4).

$$
U_{\mathrm{TM}_{2}}^{T} M_{\mathrm{TM}_{2}} U_{\mathrm{TM}_{2}}=M_{\text {diag }} \text {. }
$$

We can calculate the neutrino mixing angles from a given mixing matrix $U$ by using the following relations:

$s_{12}^{2}=\frac{\left|U_{12}\right|^{2}}{1-\left|U_{13}\right|^{2}}, \quad s_{23}^{2}=\frac{\left|U_{23}\right|^{2}}{1-\left|U_{13}\right|^{2}}, \quad$ and $\quad s_{13}^{2}=\left|U_{13}\right|^{2}$.

The mixing angles for $\mathrm{TM}_{2}$ mixing in terms of parameters $\theta$ and $\phi$ are

$$
\begin{aligned}
& s_{12}^{2}=\frac{1}{3-2 \sin ^{2} \theta}, \quad s_{13}^{2}=\frac{2}{3} \sin ^{2} \theta . \\
& s_{23}^{2}=\frac{1}{2}\left(1+\frac{\sqrt{3} \sin 2 \theta \cos \phi}{3-2 \sin ^{2} \theta}\right) .
\end{aligned}
$$

The Dirac $C P$ violating phase $\delta$ can be obtained from the Jarlskog rephasing invariant $\left(J_{C P}\right)[16]$

$$
J_{C P}=\operatorname{Im}\left(U_{11} U_{12}^{*} U_{21}^{*} U_{22}\right) .
$$

In the standard parametrization,

$$
J_{C P}=s_{12} s_{23} s_{13} c_{12} c_{23} c_{13}^{2} \sin \delta .
$$

For the $\mathrm{TM}_{2}$ mixing matrix,

$$
J_{C P}=\frac{1}{6 \sqrt{3}} \sin 2 \theta \sin \phi .
$$

From Eqs. (25) and (26), we obtain

$$
\tan \delta=\frac{\cos 2 \theta+2}{2 \cos 2 \theta+1} \tan \phi .
$$

The effective Majorana mass term relevant for neutrinoless double beta decay is given by

$$
\left|M_{e e}\right|=\left|m_{1} U_{e 1}^{2}+m_{2} e^{2 i \alpha} U_{e 2}^{2}+m_{3} e^{2 i \beta} U_{e 3}^{2}\right| .
$$

For $\mathrm{TM}_{2}$ mixing, the above expression takes the following form:

$$
\left|M_{e e}\right|=\frac{1}{3}\left|2 m_{1} \cos ^{2} \theta+m_{2} e^{2 i \alpha}+2 m_{3} e^{2 i \beta} \sin ^{2} \theta\right| .
$$

There are many ongoing and forthcoming experiments such as GERDA [17], CUORE [18], EXO [19], NEXT [20], MAJORANA [21], SuperNEMO [22], which aim to achieve a sensitivity up to $0.01 \mathrm{eV}$ for $\left|M_{e e}\right|$. Cosmological 
observations put an upper bound on the sum of neutrino masses:

$$
\Sigma=\sum_{i=1,2,3}^{3} m_{i} .
$$

Planck satellite data [23] combined with WMAP, CMB, and BAO experiments limit the sum of neutrino masses $\sum m_{i} \leq 0.23 \mathrm{eV}$ at $95 \%$ confidence level (CL). In the present work, we assume a more conservative limit of $\sum m_{i} \leq 1 \mathrm{eV}$. The existence of one texture zero in the neutrino mass matrix with $\mathrm{TM}_{2}$ mixing implies

$$
\left(M_{\mathrm{TM}_{2}}\right)_{i j}=0 .
$$

This condition yields a complex equation viz.

$$
m_{1} A+m_{2} B+m_{3} C=0,
$$

where $A=U_{a 1} U_{b 1}, B=U_{a 2} U_{b 2} e^{2 i \alpha}, C=U_{a 3} U_{b 3} e^{2 i \beta}$ and $a, b$ can take values $e, \mu$ and $\tau$. The above complex equation yields two mass ratios:

$$
\frac{m_{1}}{m_{2}}=\frac{\operatorname{Re}(C) \operatorname{Im}(B)-\operatorname{Re}(B) \operatorname{Im}(C)}{\operatorname{Re}(A) \operatorname{Im}(C)-\operatorname{Re}(C) \operatorname{Im}(A)}
$$

and

$$
\frac{m_{1}}{m_{3}}=\frac{\operatorname{Re}(C) \operatorname{Im}(B)-\operatorname{Re}(B) \operatorname{Im}(C)}{\operatorname{Re}(B) \operatorname{Im}(A)-\operatorname{Re}(A) \operatorname{Im}(B)},
$$

where $\operatorname{Re}(\operatorname{Im})$ denotes the real (imaginary) part. These mass ratios can be used to obtain the expression for the parameter $R_{\nu}$, which is the ratio of mass squared differences $\left(\Delta m_{i j}^{2}=m_{i}^{2}-m_{j}^{2}\right)$

$$
R_{\nu} \equiv \frac{\Delta m_{21}^{2}}{\left|\Delta m_{31}^{2}\right|}=\frac{\left(\frac{m_{2}}{m_{1}}\right)^{2}-1}{\left|\left(\frac{m_{3}}{m_{1}}\right)^{2}-1\right|},
$$

where $m_{1}>m_{3}$ for an inverted mass ordering (IO) and $m_{1}<m_{3}$ for the normal mass ordering (NO). For a texture zero to be compatible with the present neutrino oscillation data, the parameter $R_{\nu}$ should lie within its experimentally allowed range. The phenomenological predictions of patterns $M_{\mathrm{TM}_{2}}^{\mathrm{II}}$ and $M_{\mathrm{TM}_{2}}^{\mathrm{III}}$ are related, and one can obtain the predictions for pattern $M_{\mathrm{TM}_{2}}^{\mathrm{III}}$ by making the following transformations,

$$
\theta_{23} \rightarrow \frac{\pi}{2}-\theta_{23}, \quad \delta=\pi-\delta,
$$

on the predictions of pattern $M_{\mathrm{TM}_{2}}^{\mathrm{II}}$ and vice versa. This is because patterns $M_{\mathrm{TM}_{2}}^{\mathrm{III}}$ and $M_{\mathrm{TM}_{2}}^{\mathrm{II}}$ are related via 2-3 symmetry: $M_{\mathrm{TM}_{2}}^{\mathrm{III}}=p_{23}^{T} M_{\mathrm{TM}_{2}}^{\mathrm{II}} p_{23}$ where $p_{23}$ is the $2-3$ permutation matrix given in Eq. (19). Similarly, patterns $M_{\mathrm{TM}_{2}}^{\mathrm{IV}}$ and $M_{\mathrm{TM}_{2}}^{\mathrm{VI}}$ are related to each other by above transformations. Thus, we need to study in detail only one of the 2-3 symmetry related patterns.

In the numerical analysis, the neutrino mass matrix is reconstructed using Eq. (5), which takes into account the constraint of $\mathrm{TM}_{2}$ mixing. For the numerical analysis we generate $10^{8}$ points $\left(10^{10}\right.$ for pattern $M_{\mathrm{TM}_{2}}^{\mathrm{V}}$ with NO). The mass squared differences $\Delta m_{21}^{2}$ and $\left|\Delta m_{31}^{2}\right|$ are varied randomly within their $3 \sigma$ experimental ranges. Parameters $\theta, \phi, \alpha$ and $\beta$ are varied randomly within their full possible ranges. The texture zero constraint is imposed by requiring that the parameter $R_{\nu}$ in Eq. (35), written in terms of mass ratios $\frac{m_{1}}{m_{2}}$ and $\frac{m_{1}}{m_{3}}$ should lie within its $3 \sigma$ experimental range. In addition to above constraints, we require the allowed points to lie within the $3 \sigma$ experimental ranges of mixing angles $\theta_{12}, \theta_{13}$ and $\theta_{23}$ where the neutrino mixing angles are extracted by using the relations given in Eq. (22). The experimental ranges of various neutrino oscillation parameters with their $1,2,3 \sigma$ ranges are given in Table II.

The numerical results for one texture zero in the neutrino

\begin{tabular}{|c|c|c|c|c|}
\hline \multirow[b]{2}{*}{ Parameter } & \multicolumn{2}{|c|}{ Normal ordering } & \multicolumn{2}{|c|}{ Inverted ordering } \\
\hline & best fit $\pm 1 \sigma, \pm 2 \sigma$ & $3 \sigma$ range & best fit $\pm 1 \sigma, \pm 2 \sigma$ & $3 \sigma$ range \\
\hline$\overline{\theta_{12}^{\circ}}$ & $34.5_{-1.0,-2.0}^{+1.1,+2.3}$ & $31.5-38.0$ & $34.5_{-1.0,-2.0}^{+1.1,+2.3}$ & $31.5-38.0$ \\
\hline$\theta_{23}^{\circ}$ & $41.0_{-1.1,-1.9}^{+1.1,+2.7}$ & $38.3-52.8$ & $50.5_{-1.0,-2.3}^{+1.0,+1.7}$ & $38.5-53.0$ \\
\hline$\theta_{13}^{\circ}$ & $8.44_{-0.15,-0.34}^{+0.18,+0.26}$ & 7.9-8.9 & $8.41_{-0.17,-0.41}^{+0.16,+0.29}$ & $7.9-8.93$ \\
\hline$\delta_{C P}^{\circ}$ & $252_{-36,-99}^{+56,+99}$ & $0.0-360$ & $259_{-41,-77}^{+47,+88}$ & $0-31 \oplus 142-360$ \\
\hline$\Delta m_{21}^{2} / 10^{-5} \mathrm{eV}^{2}$ & $\begin{array}{c}7.56_{-0.19,-0.36}^{+0.19,+0.39} \\
\text { - }\end{array}$ & $7.05-8.14$ & $7.56_{-0.19,-0.36}^{+0.19,+0.39}$ & $7.05-8.14$ \\
\hline$\left|\Delta m_{3 l}^{2}\right| / 10^{-3} \mathrm{eV}^{2}$ & $2.55_{-0.04,-0.08}^{+0.04,+0.08}$ & $2.43-2.67$ & $2.49_{-0.04,-0.08}^{+0.04,+0.08}$ & $2.37-2.61$ \\
\hline
\end{tabular}
mass matrix with $\mathrm{TM}_{2}$ mixing are presented below. The main observations are

(i) All the six possible patterns of one texture zero in the neutrino mass matrix with $\mathrm{TM}_{2}$ mixing are consistent with the present neutrino oscillation data.

TABLE II. Current neutrino oscillation parameters from global fits [24]. 
(ii) The pattern $M_{\mathrm{TM}_{2}}^{\mathrm{I}}$ is consistent with normal mass ordering only.

(iii) All the viable patterns except $M_{\mathrm{TM}_{2}}^{\mathrm{I}}$, allow a quasidegenerate mass spectrum.

(iv) In case of $\mathrm{NO}$, the parameter $\left|M_{e e}\right|$ can vanish for patterns $M_{\mathrm{TM}_{2}}^{\mathrm{I}}, M_{\mathrm{TM}_{2}}^{\mathrm{II}}$ and $M_{\mathrm{TM}_{2}}^{\mathrm{III}}$. For the remaining patterns, $\left|M_{e e}\right|$ is found to be bounded from below.

(v) The smallest neutrino mass cannot vanish except for patterns $M_{\mathrm{TM}_{2}}^{\mathrm{II}}$ and $M_{\mathrm{TM}_{2}}^{\mathrm{III}}$ with IO.

(vi) The parameter $J_{C P}$ cannot vanish for patterns $M_{\mathrm{TM}_{2}}^{\mathrm{II}}$ with IO and $M_{\mathrm{TM}_{2}}^{\mathrm{V}}$ with $\mathrm{NO}$, implying that these patterns are necessarily $C P$ violating.

(vii) The atmospheric neutrino mixing angle $\theta_{23}$ remains below (above) $45^{\circ}$ for pattern $M_{\mathrm{TM}_{2}}^{\mathrm{IV}}\left(M_{\mathrm{TM}_{2}}^{\mathrm{VI}}\right)$ with NO.

The numerical predictions for the presently unknown neutrino parameters have been summarized in Table III. The allowed ranges (at $3 \sigma \mathrm{CL}$ ) of parameters $\theta_{12}, \theta$ and $J_{C P}$ are $\left(35.65^{\circ}-35.76^{\circ}\right),\left(9.68^{\circ}-10.93^{\circ}\right)$ and $(-0.0363-0.0363)$, respectively, for all the allowed patterns except for patterns $M_{\mathrm{TM}_{2}}^{\mathrm{II}, \mathrm{III}}$ with IO and $M_{\mathrm{TM}_{2}}^{\mathrm{V}}$ with $\mathrm{NO}$, for which $J_{C P}$ cannot vanish and has the allowed ranges $\pm(0.006-0.036)$ and $\pm(0.0165-0.036)$, respectively. Some of the interesting correlation plots are given in Figs. 1, 2, and 3. Figure 1(a) shows that the Majorana phases $\alpha$ and $\beta$ are strongly correlated with each other for pattern $M_{\mathrm{TM}_{2}}^{\mathrm{I}}$ with NO. One can see from Fig. 1(c) that the
Dirac phase $\delta$ and phase $\phi$ are linearly correlated and are almost identical to each other. From Eq. (27) we can see that the ratio $\left(\frac{\cos 2 \theta+2}{2 \cos 2 \theta+1}\right)$ multiplying with $\tan \phi$ remains $\approx 1$ for the allowed values of $\theta$. This leads to the feature $\delta \approx \phi$, for all the neutrino mass matrices with a texture zero and $\mathrm{TM}_{2}$ mixing.

For pattern $M_{\mathrm{TM}_{2}}^{\mathrm{II}}$, the Dirac phase $\delta$ is restricted to two regions [Fig. 2(a)]. The correlation between mixing angles $\theta_{13}$ and $\theta_{12}$ is shown in Fig. 2(c). This is a generic feature of $\mathrm{TM}_{2}$ mixing arising from Eq. (23). Since the TBM value of $\theta_{12}$ is already above its experimental best-fit value, an increase in $\theta$ drives $\theta_{12}$ further away from the best-fit experimental value. Thus, $\mathrm{TM}_{2}$ mixing leads to some tension with mixing angle $\theta_{12}$. Figure 3 shows the 2-3 symmetry between patterns $M_{\mathrm{TM}_{2}}^{\mathrm{IV}}$ and $M_{\mathrm{TM}_{2}}^{\mathrm{VI}}$.

\section{Symmetry realization}

The neutrino mass matrices with one texture zero and $\mathrm{TM}_{2}$ mixing can be realized in the framework of type-I+II seesaw mechanism $[25,26]$ using $A_{4}$ [27] symmetry. In addition to the three standard model lepton $S U(2)_{L}$ doublets $D_{l_{L}}$ (where $l=e, \mu$ and $\tau$ ) and three right-handed charged lepton singlets $l_{R}$, we need a $S U(2)_{L}$ singlet right-handed neutrino $\nu_{R}$, six $S U(2)_{L}$ doublet Higgs fields $\psi_{i}$ and $\varphi_{i}(i=1,2,3)$, and two $S U(2)_{L}$ triplet Higgs fields $\Delta_{1}, \Delta_{2}$. We also impose an additional $Z_{2}$ symmetry, to prevent couplings between charged leptons (neutrinos) and
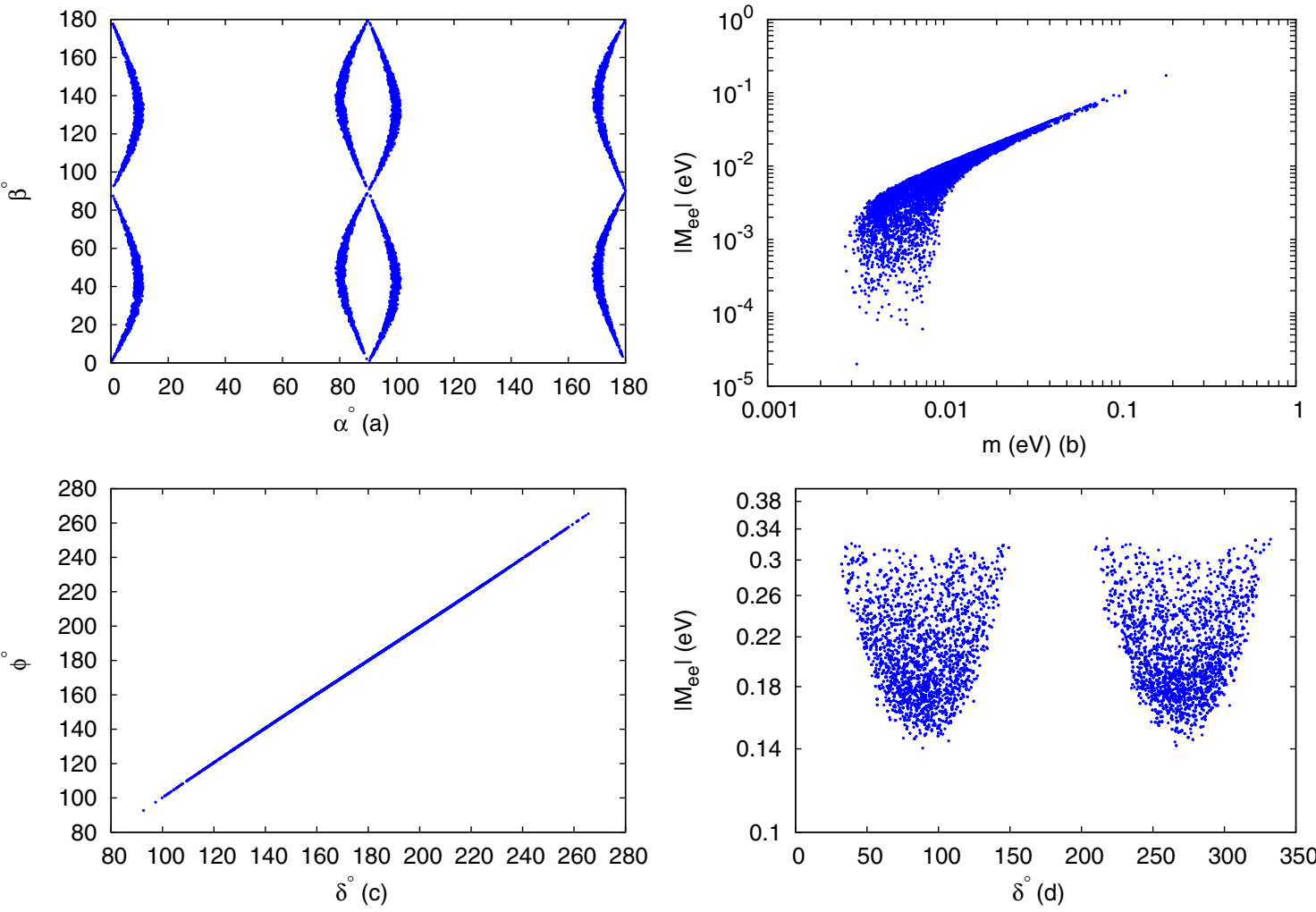

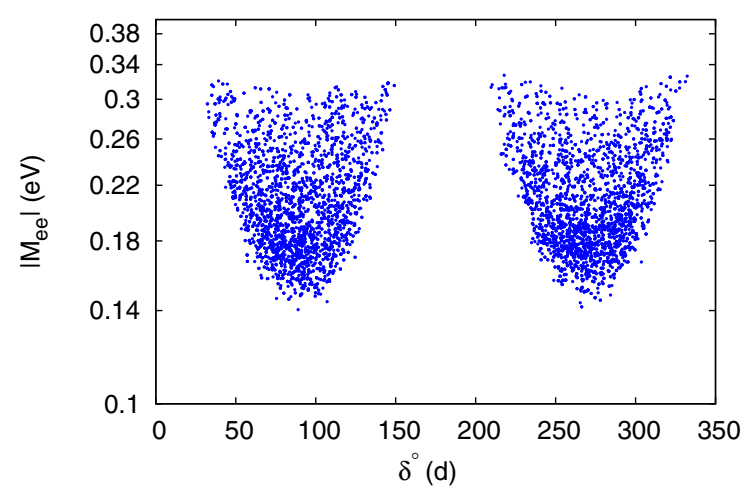

FIG. 1. Correlation plots for patterns $M_{\mathrm{TM}_{2}}^{\mathrm{I}}$ (a), $M_{\mathrm{TM}_{2}}^{\mathrm{II}}$ (b), $M_{\mathrm{TM}_{2}}^{\mathrm{IV}}$ (c) and $M_{\mathrm{TM}_{2}}^{\mathrm{V}}$ (d) with NO. 

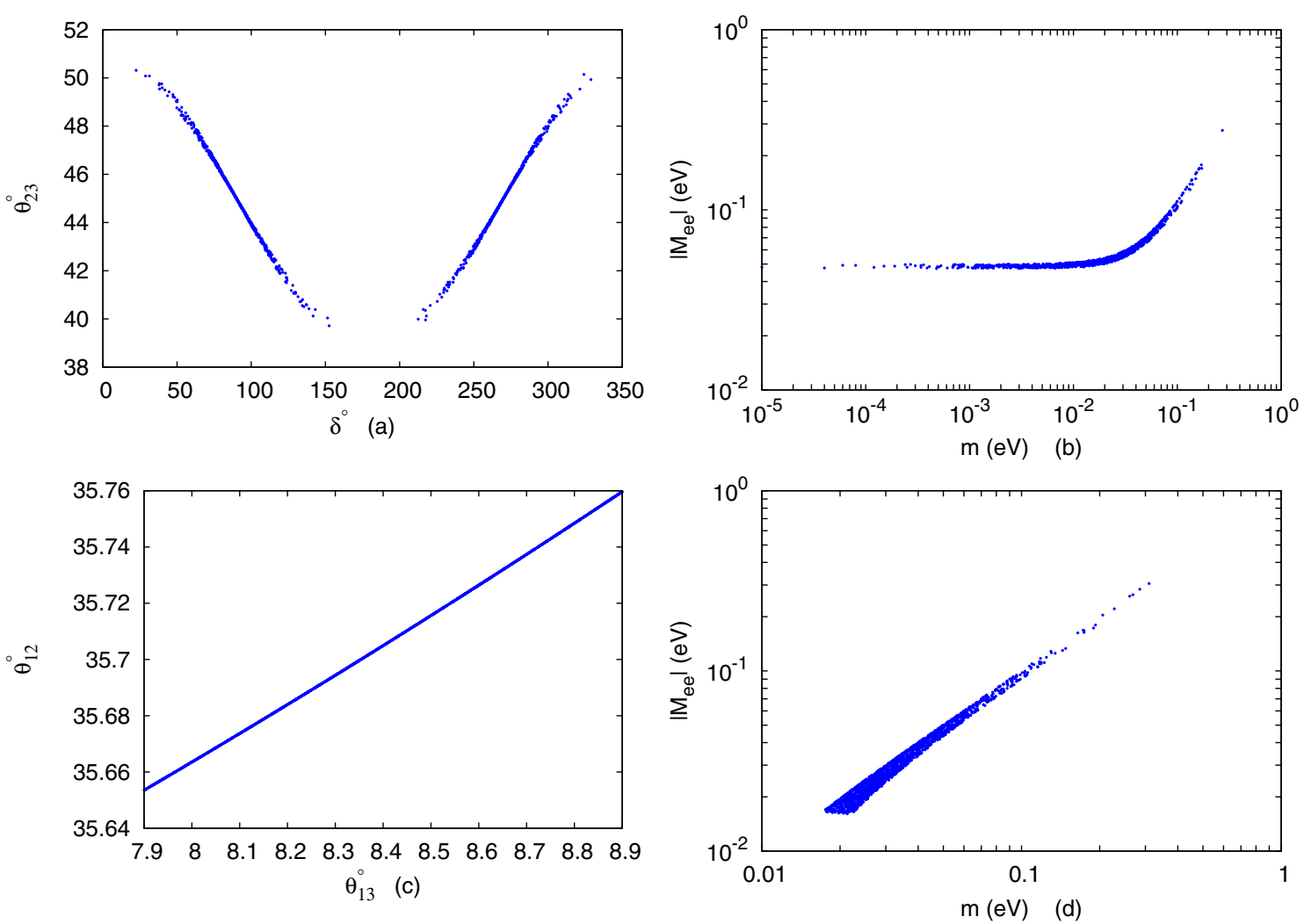

FIG. 2. Correlation plots for patterns $M_{\mathrm{TM}_{2}}^{\mathrm{II}}$ (a,b), $M_{\mathrm{TM}_{2}}^{\mathrm{IV}}$, (c) and $M_{\mathrm{TM}_{2}}^{\mathrm{V}}$ (d) with IO.

scalars $\varphi_{i}\left(\psi_{i}\right)$. Below we discuss in detail the symmetry realization of pattern $M_{\mathrm{TM}_{2}}^{\mathrm{I}}$. The transformation properties of various fields under $A_{4}$ and $Z_{2}$ corresponding to pattern I are given in Table IV. These transformation properties lead to the following Yukawa Lagrangian which is invariant under $A_{4}$ and $Z_{2}$ :

$$
\begin{aligned}
-\mathcal{L}_{\text {Yukawa }}= & y_{1}\left(\bar{D}_{e_{L}} \psi_{1}+\bar{D}_{\mu_{L}} \psi_{2}+\bar{D}_{\tau_{L}} \psi_{3}\right)_{\underline{1}} e_{R_{1}}+y_{2}\left(\bar{D}_{e_{L}} \psi_{1}+\omega^{2} \bar{D}_{\mu_{L}} \psi_{2}+\omega \bar{D}_{\tau_{L}} \psi_{3}\right)_{\underline{1}^{\prime}} \tau_{R_{\underline{1}^{\prime \prime}}} \\
& +y_{3}\left(\bar{D}_{e_{L}} \psi_{1}+\omega \bar{D}_{\mu_{L}} \psi_{2}+\omega^{2} \bar{D}_{\tau_{L}} \psi_{3}\right)_{\underline{1}^{\prime \prime}} \mu_{R_{\underline{1}^{\prime}}}+y_{4}\left(\bar{D}_{e_{L}} \tilde{\varphi}_{1}+\bar{D}_{\mu_{L}} \tilde{\varphi}_{2}+\bar{D}_{\tau_{L}} \tilde{\varphi}_{3}\right)_{\underline{1}} \nu_{R_{\underline{1}}} \\
& -y_{\Delta_{1}}\left(D_{e_{L}}^{T} C^{-1} D_{e_{L}}+\omega D_{\mu_{L}}^{T} C^{-1} D_{\mu_{L}}+\omega^{2} D_{\tau_{L}}^{T} C^{-1} D_{\tau_{L}}\right)_{\underline{1}^{\prime \prime}} i \tau_{2} \Delta_{1_{\underline{1}^{\prime}}} \\
& -y_{\Delta_{2}}\left(D_{e_{L}}^{T} C^{-1} D_{e_{L}}+\omega^{2} D_{\mu_{L}}^{T} C^{-1} D_{\mu_{L}}+\omega D_{\tau_{L}}^{T} C^{-1} D_{\tau_{L}}\right)_{\underline{1}^{\prime}} i \tau_{2} \Delta_{\underline{1}_{\underline{1}^{\prime \prime}}}-m_{R}\left(\nu_{R}^{T} C^{-1} \nu_{R}\right)+\text { H.c. },
\end{aligned}
$$

where $\tilde{\varphi}=i \tau_{2} \varphi^{*}$. Assuming that $\psi_{i}$ Higgs fields acquire nonzero vacuum expectation values (VEVs) along the direction $\langle\psi\rangle_{o}=v_{\psi}(1,1,1)^{T}$ leads to the following form of the charged lepton mass matrix:
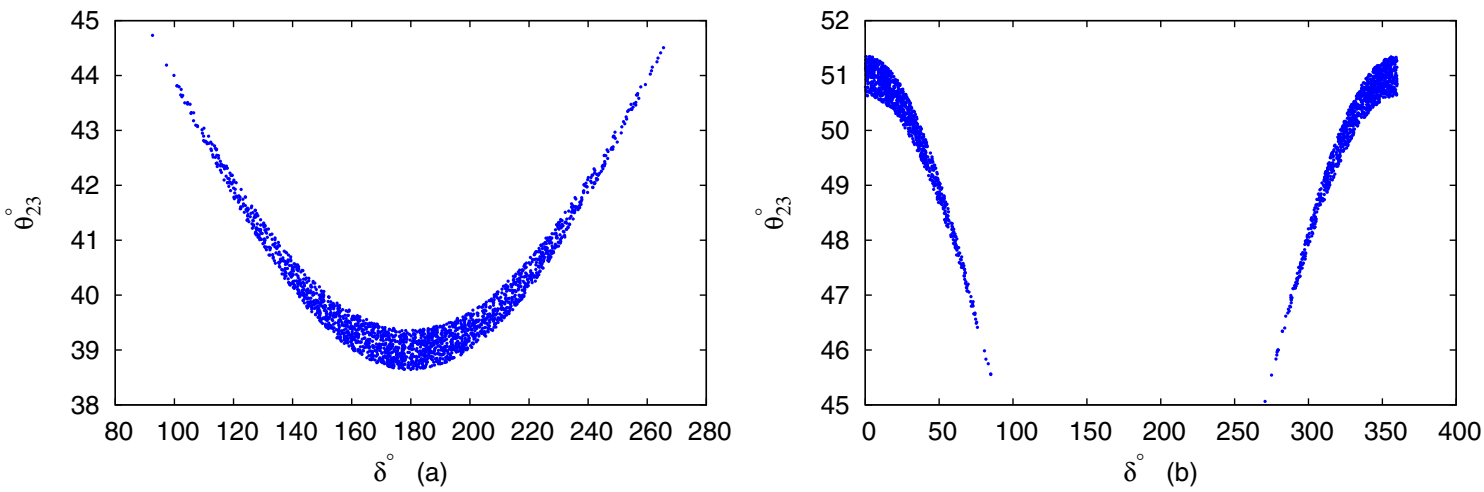

FIG. 3. Correlation plots for patterns $M_{\mathrm{TM}_{2}}^{\mathrm{IV}}$ (a) and $M_{\mathrm{TM}_{2}}^{\mathrm{VI}}$ (b) with NO. 
TABLE III. Numerical predictions (at $3 \sigma \mathrm{CL}$ ) for patterns having one texture zero in $M_{\nu}$ with $\mathrm{TM}_{2}$ mixing.

\begin{tabular}{lccccc}
\hline \hline Pattern & Mass ordering & $\left|M_{e e}\right|(\mathrm{eV})$ & Lightest neutrino mass $m(\mathrm{eV})$ & $\sum m_{i}(\mathrm{eV})$ & $\delta^{\circ}$ \\
\hline I & NO & 0.0 & $0.0026-0.0084$ & $0.061-0.073$ & $0-360$ \\
II & NO & $0.0-0.172$ & $0.002-0.183$ & $0.056-0.57$ & $0-360$ \\
& IO & $0.047-0.275$ & $0.0-0.272$ & $0.097-0.825$ & $21-154 \oplus 200-345$ \\
III & NO & $0.0-0.172$ & $0.002-0.183$ & $0.056-0.57$ & $0-360$ \\
& IO & $0.047-0.275$ & $0.0-0.272$ & $0.097-0.825$ & $29-159 \oplus 200-332$ \\
IV & NO & $0.034-0.237$ & $0.035-0.3$ & $0.13-0.9$ & $90-270$ \\
& IO & $0.015-0.126$ & $0.007-0.18$ & $0.106-0.553$ & $0-360$ \\
V & NO & $0.14-0.34$ & $0.147-0.332$ & $0.45-1$ & $32-150 \oplus 209-333$ \\
& IO & $0.015-0.305$ & $0.017-0.31$ & $0.127-0.94$ & $0-360$ \\
VI & NO & $0.034-0.237$ & $0.035-0.3$ & $0.13-0.9$ & $0-90 \oplus 270-360$ \\
& IO & $0.015-0.126$ & $0.007-0.18$ & $0.106-0.553$ & $0-360$ \\
\hline \hline
\end{tabular}

$$
m_{l}=\left(\begin{array}{ccc}
y_{1} v_{\psi} & y_{2} v_{\psi} & y_{3} v_{\psi} \\
y_{1} v_{\psi} & y_{2} \omega v_{\psi} & y_{3} \omega^{2} v_{\psi} \\
y_{1} v_{\psi} & y_{2} \omega^{2} v_{\psi} & y_{3} \omega v_{\psi}
\end{array}\right) .
$$

The $\varphi_{i}$ fields which couple to neutrinos are assumed to have the VEV alignment: $\langle\varphi\rangle_{o}=v_{\varphi}(0,-1,1)^{T}$. Similar vacuum alignment has been obtained earlier in Ref. [28] for $A_{4}$ and $S U(2)_{L}$ triplet scalars. The above VEV alignment leads to the following Dirac neutrino mass matrix:

$$
m_{D}=y_{4} v_{\varphi}(0,-1,1)^{T} .
$$

With only one right-handed neutrino with mass $m_{R}$, the effective neutrino mass matrix obtained using the type-I seesaw mechanism $m_{\nu}^{I} \approx m_{D} m_{R}^{-1} m_{D}^{T}$, has the form

$$
m_{\nu}^{I}=a\left(\begin{array}{ccc}
0 & 0 & 0 \\
0 & 1 & -1 \\
0 & -1 & 1
\end{array}\right) \quad \text { where } a=y_{4}^{2} v_{\varphi}^{2} / m_{R}
$$

The type-II seesaw contribution to the effective neutrino mass matrix has the following form which is obtained when the $S U(2)_{L}$ triplet Higgses $\Delta_{1}$ and $\Delta_{2}$ acquire nonzero and small VEVs:

$$
m_{\nu}^{\mathrm{II}}=\left(\begin{array}{ccc}
b+c & 0 & 0 \\
0 & \omega b+\omega^{2} c & 0 \\
0 & 0 & \omega^{2} b+\omega c
\end{array}\right),
$$

where $b=y_{\Delta_{1}} v_{\Delta_{1}}$ and $c=y_{\Delta_{2}} v_{\Delta_{2}}$. The collective effective neutrino mass matrix $m_{\nu}=m_{\nu}^{\mathrm{I}}+m_{\nu}^{\mathrm{II}}$ from the type-I + II

\begin{tabular}{|c|c|c|c|c|c|c|c|c|c|c|c|}
\hline Pattern & Symmetry & $D_{l_{L}}$ & $e_{R}$ & $\mu_{R}$ & $\tau_{R}$ & $\nu_{R}$ & $\psi$ & $\varphi$ & $\Delta_{1}$ & $\Delta_{2}$ & $D_{L}$ triplet representation under $A_{4}$ \\
\hline \multirow{3}{*}{$M_{\mathrm{TM}_{2}}^{\mathrm{I}}$} & $S U(2)_{L}$ & 2 & 1 & 1 & 1 & 1 & 2 & 2 & 2 & 3 & \multirow{3}{*}{$3 \sim$} \\
\hline & $A_{4}$ & 3 & 1 & $1^{\prime}$ & $1^{\prime \prime}$ & 1 & 3 & 3 & $1^{\prime}$ & $1^{\prime \prime}$ & \\
\hline & $Z_{2}$ & 1 & 1 & 1 & 1 & -1 & 1 & -1 & 1 & 1 & \\
\hline \multirow{3}{*}{$M_{\mathrm{TM}_{2}}^{\mathrm{II}}$} & $S U(2)_{L}$ & 2 & 1 & 1 & 1 & 1 & 2 & 2 & 3 & 3 & $D_{e_{L}}$ \\
\hline & $A_{4}$ & 3 & 1 & $1^{\prime}$ & $1^{\prime \prime}$ & 1 & 3 & 3 & 1 & $1^{\prime \prime}$ & $3 \sim D_{\mu_{L}}$ \\
\hline & $Z_{2}$ & 1 & 1 & 1 & 1 & -1 & 1 & -1 & 1 & 1 & $D_{\tau_{L}}$ \\
\hline \multirow{3}{*}{$M_{\mathrm{TM}_{2}}^{\mathrm{III}}$} & $S U(2)_{L}$ & 2 & 1 & 1 & 1 & 1 & 2 & 2 & 3 & 3 & \multirow[b]{2}{*}{$3 \sim$} \\
\hline & $A_{4}$ & 3 & 1 & $1^{\prime}$ & $1^{\prime \prime}$ & 1 & 3 & 3 & 1 & $1^{\prime}$ & \\
\hline & $Z_{2}$ & 1 & 1 & 1 & 1 & -1 & 1 & -1 & 1 & 1 & $D_{\tau_{L}}$ \\
\hline \multirow{3}{*}{$M_{\mathrm{TM}_{2}}^{\mathrm{IV}}$} & $S U(2)_{L}$ & 2 & 1 & 1 & 1 & 1 & 2 & 2 & 3 & 3 & $D_{\mu_{L}}$ \\
\hline & $A_{4}$ & 3 & $1^{\prime \prime}$ & 1 & $1^{\prime}$ & 1 & 3 & 3 & $1^{\prime}$ & $1^{\prime \prime}$ & $D_{\tau_{L}}$ \\
\hline & $Z_{2}$ & 1 & 1 & 1 & 1 & -1 & 1 & -1 & 1 & 1 & $D_{e_{L}}$ \\
\hline \multirow{3}{*}{$M_{\mathrm{TM}_{2}}^{\mathrm{V}}$} & $S U(2)_{L}$ & 2 & 1 & 1 & 1 & 1 & 2 & 2 & 3 & 3 & $D_{\tau_{L}}$ \\
\hline & $A_{4}$ & 3 & $1^{\prime}$ & $1^{\prime \prime}$ & 1 & 1 & 3 & 3 & 1 & $1^{\prime}$ & $3 \sim\left(D_{e_{L}}\right.$ \\
\hline & $Z_{2}$ & 1 & 1 & 1 & 1 & -1 & 1 & -1 & 1 & 1 & $D_{\mu_{L}}$ \\
\hline \multirow{3}{*}{$M_{\mathrm{TM}_{2}}^{\mathrm{VI}}$} & $S U(2)_{L}$ & 2 & 1 & 1 & 1 & 1 & 2 & 2 & 3 & 3 & \multirow[b]{2}{*}{$3 \sim$} \\
\hline & $A_{4}$ & 3 & $1^{\prime}$ & $1^{\prime \prime}$ & 1 & 1 & 3 & 3 & $1^{\prime \prime}$ & $1^{\prime}$ & \\
\hline & $Z_{2}$ & 1 & 1 & 1 & 1 & -1 & 1 & -1 & 1 & 1 & $D_{\mu_{L}}$ \\
\hline
\end{tabular}
seesaw mechanism becomes

TABLE IV. Transformation properties of various fields under $A_{4}$ and $Z_{2}$. The VEV alignments for $\psi$ and $\varphi$ are $\langle\psi\rangle_{o}=v_{\psi}(1,1,1)^{T}$ and $\langle\varphi\rangle_{o}=v_{\varphi}(0,-1,1)^{T}$. 
$m_{\nu}=\left(\begin{array}{ccc}b+c & 0 & 0 \\ 0 & a+\omega b+\omega^{2} c & -a \\ 0 & -a & a+\omega^{2} b+\omega c\end{array}\right)$.

In the present basis, the charged lepton mass matrix is nondiagonal. We move to the diagonal charged lepton mass matrix basis using the transformation $M_{l}=U_{L}^{\dagger} m_{l} U_{R}$, where

$$
U_{L}=\frac{1}{3}\left(\begin{array}{ccc}
1 & 1 & 1 \\
1 & \omega & \omega^{2} \\
1 & \omega^{2} & \omega
\end{array}\right)
$$

and $U_{R}$ is a unit matrix. In this basis, the effective neutrino mass matrix becomes

$$
M_{\nu}=\left(\begin{array}{ccc}
0 & b & c \\
b & -a+c & a \\
c & a & -a+b
\end{array}\right)
$$

which is the patten $M_{\mathrm{TM}_{2}}^{\mathrm{I}}$ of one texture zero with $\mathrm{TM}_{2}$ mixing. The symmetry realization of other patterns can be obtained in a similar way. We have summarized the desired transformation properties of various leptonic and scalar fields (under $A_{4}$ and $Z_{2}$ ) which lead to neutrino mass matrices with one texture zero and $\mathrm{TM}_{2}$ mixing, in Table IV.

For the symmetry realization of above patterns, we require many Higgs $S U(2)_{L}$ doublets. It should be noted that such multi-Higgs models generally lead to flavorchanging neutral currents which can contribute to charged lepton flavor violating decays. However, an explicit calculation of such effects is beyond the scope of the present work.

\section{TM 1 MIXING AND ONE TEXTURE ZERO}

\section{A. $\mathbf{T M}_{1}$ mixing}

In section II we studied the one texture zero patterns having $\mathrm{TM}_{2}$ mixing. For these patterns the allowed values of $\theta_{12}$ lie in the $2 \sigma$ upper limit and values within the $1 \sigma$ experimental range are not allowed. This leads to some tension with the present neutrino oscillation data. However, this is a generic feature of $\mathrm{TM}_{2}$ mixing. One possible way to resolve this discrepancy is to consider charged lepton corrections to the neutrino mixing matrix.

Alternatively, instead of considering $\mathrm{TM}_{2}$ mixing one may also consider $\mathrm{TM}_{1}$ mixing where $\theta_{12}$ can take values which are in good agreement with the present neutrino oscillation data. In this section we explore the neutrino mass matrices having one texture zero along with $\mathrm{TM}_{1}$ mixing. The $\mathrm{TM}_{1}$ mixing matrix can be parametrized as $[4,5,12,14]$
$U_{\mathrm{TM}_{1}}=\left(\begin{array}{ccc}\sqrt{\frac{2}{3}} & \frac{1}{\sqrt{3}} \cos \theta & \frac{1}{\sqrt{3}} \sin \theta \\ -\frac{1}{\sqrt{6}} & \frac{1}{\sqrt{3}} \cos \theta-\frac{e^{i \phi} \sin \theta}{\sqrt{2}} & \frac{1}{\sqrt{3}} \sin \theta+\frac{e^{i \phi} \cos \theta}{\sqrt{2}} \\ -\frac{1}{\sqrt{6}} & \frac{1}{\sqrt{3}} \cos \theta+\frac{e^{i \phi} \sin \theta}{\sqrt{2}} & \frac{1}{\sqrt{3}} \sin \theta-\frac{e^{i \phi} \cos \theta}{\sqrt{2}}\end{array}\right)$

here, the first column of the neutrino mixing matrix is identical with TBM mixing matrix and the other two columns have been parametrized in terms of two free parameters $(\theta$ and $\phi)$ after taking into consideration the unitarity constraints on the mixing matrix. The corresponding neutrino mass matrix for $\mathrm{TM}_{1}$ mixing is given as

$$
M_{\mathrm{TM}_{1}}=U_{\mathrm{TM}_{1}}^{*} M_{\mathrm{diag}} U_{\mathrm{TM}_{1}}^{\dagger} .
$$

\section{B. One zero in $\boldsymbol{M}_{\mathrm{TM}_{1}}$}

A neutrino mass matrix with $\mathrm{TM}_{1}$ mixing can be written as

$M_{\mathrm{TM}_{1}}=\left(\begin{array}{ccc}a & 2 b & 2 c \\ 2 b & 4 b+d & a-b-c-d \\ 2 c & a-b-c-d & 4 c+d\end{array}\right)$.

All the neutrino mass matrices with one texture zero and $\mathrm{TM}_{1}$ mixing can be obtained by substituting the respective constraints from Table I in Eq. (47):

$$
\begin{aligned}
M_{\mathrm{TM}_{1}}^{\mathrm{I}} & =\left(\begin{array}{ccc}
0 & 2 b & 2 c \\
2 b & 4 b+d & -b-c-d \\
2 c & -b-c-d & 4 c+d
\end{array}\right) \\
M_{\mathrm{TM}_{1}}^{\mathrm{II}} & =\left(\begin{array}{ccc}
a & 0 & 2 c \\
0 & d & a-c-d \\
2 c & a-c-d & 4 c+d
\end{array}\right) \\
M_{\mathrm{TM}_{1}}^{\mathrm{III}} & =\left(\begin{array}{ccc}
a & 2 b & 0 \\
2 b & 4 b+d & a-b-d \\
0 & a-b-d & d
\end{array}\right) \\
M_{\mathrm{TM}_{1}}^{\mathrm{IV}_{1}} & =\left(\begin{array}{ccc}
a & 2 b & 2 c \\
2 b & 0 & a+3 b-c \\
2 c & a+3 b-c & 4 c-4 b
\end{array}\right) \\
M_{\mathrm{TM}_{1}}^{\mathrm{V}} & =\left(\begin{array}{ccc}
a & 2 b & 2 c \\
2 b & a+3 b-c & 0 \\
2 c & 0 & a+3 c-b
\end{array}\right)
\end{aligned}
$$




$$
M_{\mathrm{TM}_{1}}^{\mathrm{VI}}=\left(\begin{array}{ccc}
a & 2 b & 2 c \\
2 b & 4 b-4 c & a+3 c-b \\
2 c & a+3 c-b & 0
\end{array}\right) .
$$

A neutrino mass matrix with $\mathrm{TM}_{1}$ mixing can be diagonalized by the mixing matrix $U=U_{\mathrm{TM}_{1}}$ given in Eq. (45),

$$
U_{\mathrm{TM}_{1}}^{T} M_{\mathrm{TM}_{1}} U_{\mathrm{TM}_{1}}=M_{\mathrm{diag}} .
$$

The mixing angles for $\mathrm{TM}_{1}$ mixing in terms of parameters $\theta$ and $\phi$ are

$$
\begin{aligned}
& s_{13}^{2}=\frac{1}{3} \sin ^{2} \theta, \quad s_{12}^{2}=1-\frac{2}{3-\sin ^{2} \theta}, \\
& s_{23}^{2}=\frac{1}{2}\left(1+\frac{\sqrt{6} \sin 2 \theta \cos \phi}{3-\sin ^{2} \theta}\right) .
\end{aligned}
$$

We see from Eq. (55) that the solar mixing angle $\theta_{12}$ is smaller than its TBM value $s_{12}^{2}=1 / 3$. In contrast, for $\mathrm{TM}_{2}$ mixing, the value of $\theta_{12}$ is larger than the TBM value. Since the experimental best-fit value of $\theta_{12}$ is towards the lower side of the TBM value, $\mathrm{TM}_{1}$ mixing is more appealing than $\mathrm{TM}_{2}$ mixing. The Dirac $C P$ violating phase $\delta$ can be obtained from the Jarlskog rephasing invariant $\left(J_{C P}\right)[16]$ :

$$
J_{C P}=\operatorname{Im}\left(U_{11} U_{12}^{*} U_{21}^{*} U_{22}\right)
$$

For the $\mathrm{TM}_{1}$ mixing matrix,

$$
J_{C P}=\frac{1}{6 \sqrt{6}} \sin 2 \theta \sin \phi .
$$

Using Eqs. (25) and (57), we obtain

$$
\tan \delta=\frac{\cos 2 \theta+5}{5 \cos 2 \theta+1} \tan \phi .
$$

The effective Majorana mass for $\mathrm{TM}_{1}$ mixing is given by

$$
\left|M_{e e}\right|=\frac{1}{3}\left|2 m_{1}+m_{2} e^{2 i \alpha} \cos ^{2} \theta+m_{3} e^{2 i \beta} \sin ^{2} \theta\right| .
$$

The existence of one texture zero in the neutrino mass matrix implies

$$
\left(M_{\mathrm{TM}_{1}}\right)_{i j}=0 \text {. }
$$

Following the same procedure as we did for $\mathrm{TM}_{2}$ mixing, we analyze the phenomenological predictions of neutrino mass matrices having one texture zero and $\mathrm{TM}_{1}$ mixing.

The main results of the numerical analysis are

(i) All six patterns of one texture zero in the neutrino mass matrix with $\mathrm{TM}_{1}$ mixing are consistent with the present neutrino oscillation data.

(ii) The pattern $M_{\mathrm{TM}_{1}}^{\mathrm{I}}$ is consistent with normal mass ordering only.

(iii) All the allowed patterns except for $M_{\mathrm{TM}_{1}}^{\mathrm{I}}$, allow a quasidegenerate mass spectrum.

(iv) In case of $\mathrm{NO}$, vanishing values of the parameter $\left|M_{e e}\right|$ are allowed for patterns $M_{\mathrm{TM}_{1}}^{\mathrm{I}}, M_{\mathrm{TM}_{1}}^{\mathrm{II}}$ and $M_{\mathrm{TM}_{1}}^{\mathrm{III}}$. For the remaining patterns $\left|M_{e e}\right|$ is found to be bounded from below.

(v) The smallest neutrino mass can have vanishing values for patterns $M_{\mathrm{TM}_{1}}^{\mathrm{II}}$ and $M_{\mathrm{TM}_{1}}^{\mathrm{III}}$ with IO.

(vi) The parameter $J_{C P}$ cannot vanish for any of the allowed patterns implying that these patterns are necessarily $C P$ violating.

(vii) The atmospheric neutrino mixing angle $\theta_{23}$ remains below (above) $45^{\circ}$ for pattern $M_{\mathrm{TM}_{1}}^{\mathrm{IV}}\left(M_{\mathrm{TM}_{1}}^{\mathrm{VI}}\right)$ with NO.

Numerical results for the presently unknown neutrino parameters have been summarized in Table V. The allowed ranges (at $3 \sigma \mathrm{CL}$ ) of parameters $\theta_{12}, \theta$ and $J_{C P}$ are $\left(34.26^{\circ}-\right.$ $\left.34.48^{\circ}\right)$, $\left(13.77^{\circ}-15.55^{\circ}\right)$ and $\pm(0.022-0.035)$, respectively, for all the allowed patterns. Some of the correlation plots are given in Figs. 4 and 5. Figure 4(a) depicts the correlation plot between Dirac phase $\delta$ and mixing angle $\theta_{23}$ for patten $M_{\mathrm{TM}_{1}}^{\mathrm{II}}$ with NO. The $C P$ violating Dirac

TABLE V. Numerical predictions (at $3 \sigma \mathrm{CL}$ ) for patterns having one texture zero in $M_{\nu}$ with $\mathrm{TM}_{1}$ mixing.

\begin{tabular}{lccccc}
\hline \hline Pattern & Mass ordering & $\left|M_{e e}\right|(\mathrm{eV})$ & $m(\mathrm{eV})$ & $m_{i}(\mathrm{eV})$ & $\delta^{\circ}$ \\
\hline I & NO & 0.0 & $0.0022-0.0071$ & $0.06-0.07$ & $46-127 \oplus 233-314$ \\
II & NO & $0.0-0.124$ & $0.0017-0.124$ & $0.06-0.382$ & $46-127 \oplus 233-314$ \\
& IO & $0.047-0.258$ & $0.0-0.273$ & $0.097-0.83$ & $46-127 \oplus 233-314$ \\
III & NO & $0.0-0.124$ & $0.0017-0.124$ & $0.06-0.382$ & $46-127 \oplus 233-314$ \\
& IO & $0.047-0.258$ & $0.0-0.273$ & $0.097-0.83$ & $46-127 \oplus 233-314$ \\
IV & NO & $0.03-0.3$ & $0.033-0.3$ & $0.13-0.91$ & $90-127 \oplus 233-270$ \\
& IO & $0.017-0.24$ & $0.0037-0.28$ & $0.1-0.85$ & $45-122 \oplus 235-313$ \\
V & NO & $0.14-0.311$ & $0.15-0.331$ & $0.46-1$ & $48-125 \oplus 235-313$ \\
& IO & $0.018-0.24$ & $0.016-0.26$ & $0.12-0.78$ & $45-125 \oplus 235-314$ \\
VI & NO & $0.029-0.3$ & $0.031-0.3$ & $0.12-0.9$ & $45-90 \oplus 270-314$ \\
& IO & $0.017-0.24$ & $0.0037-0.28$ & $0.1-0.85$ & $52-125 \oplus 235-314$ \\
\hline \hline
\end{tabular}



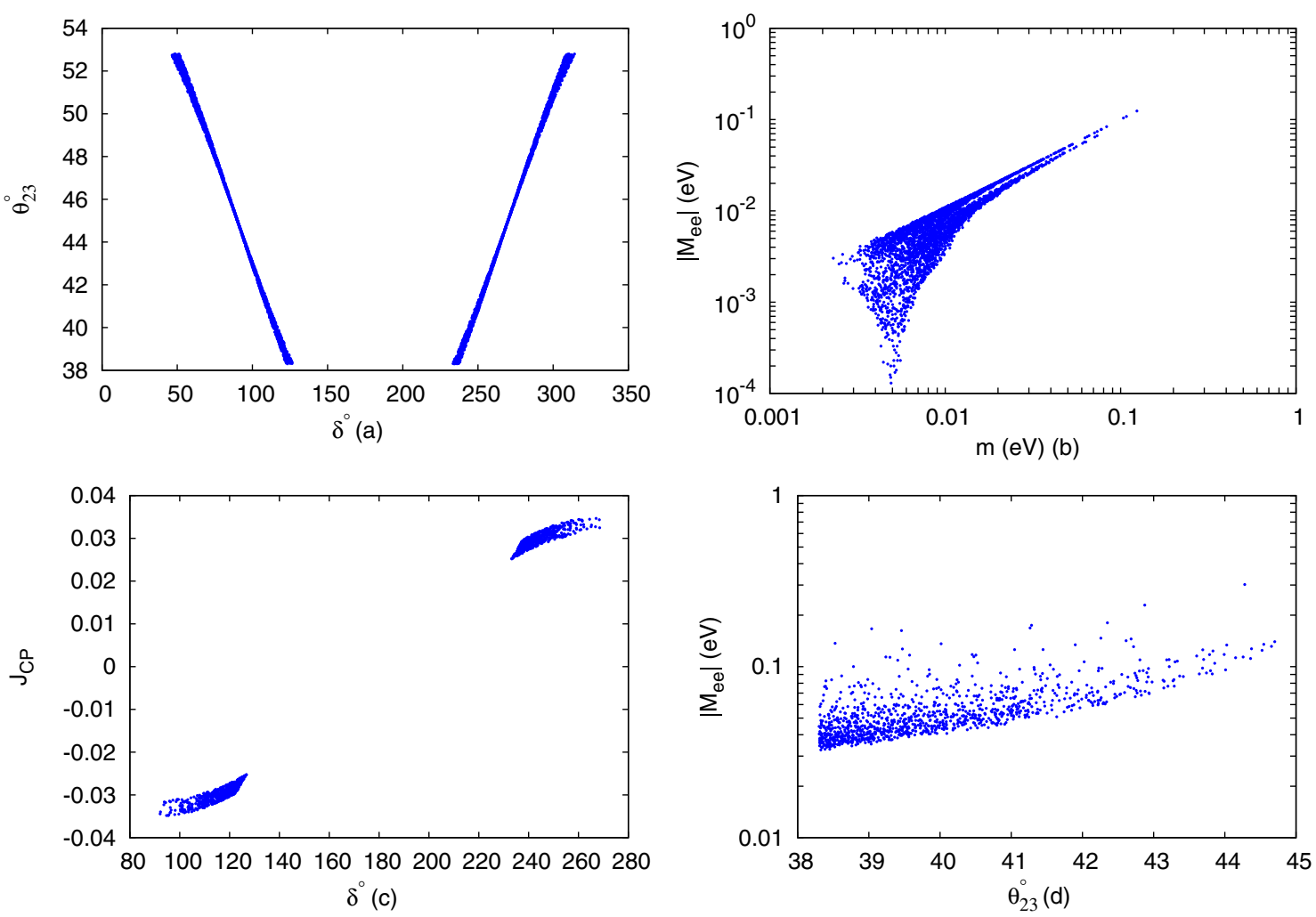

FIG. 4. Correlation plots for patterns $M_{\mathrm{TM}_{1}}^{\mathrm{I}}$ (a), $M_{\mathrm{TM}_{1}}^{\mathrm{II}}$ (b), and $M_{\mathrm{TM}_{1}}^{\mathrm{IV}}$ (c),(d) with NO.
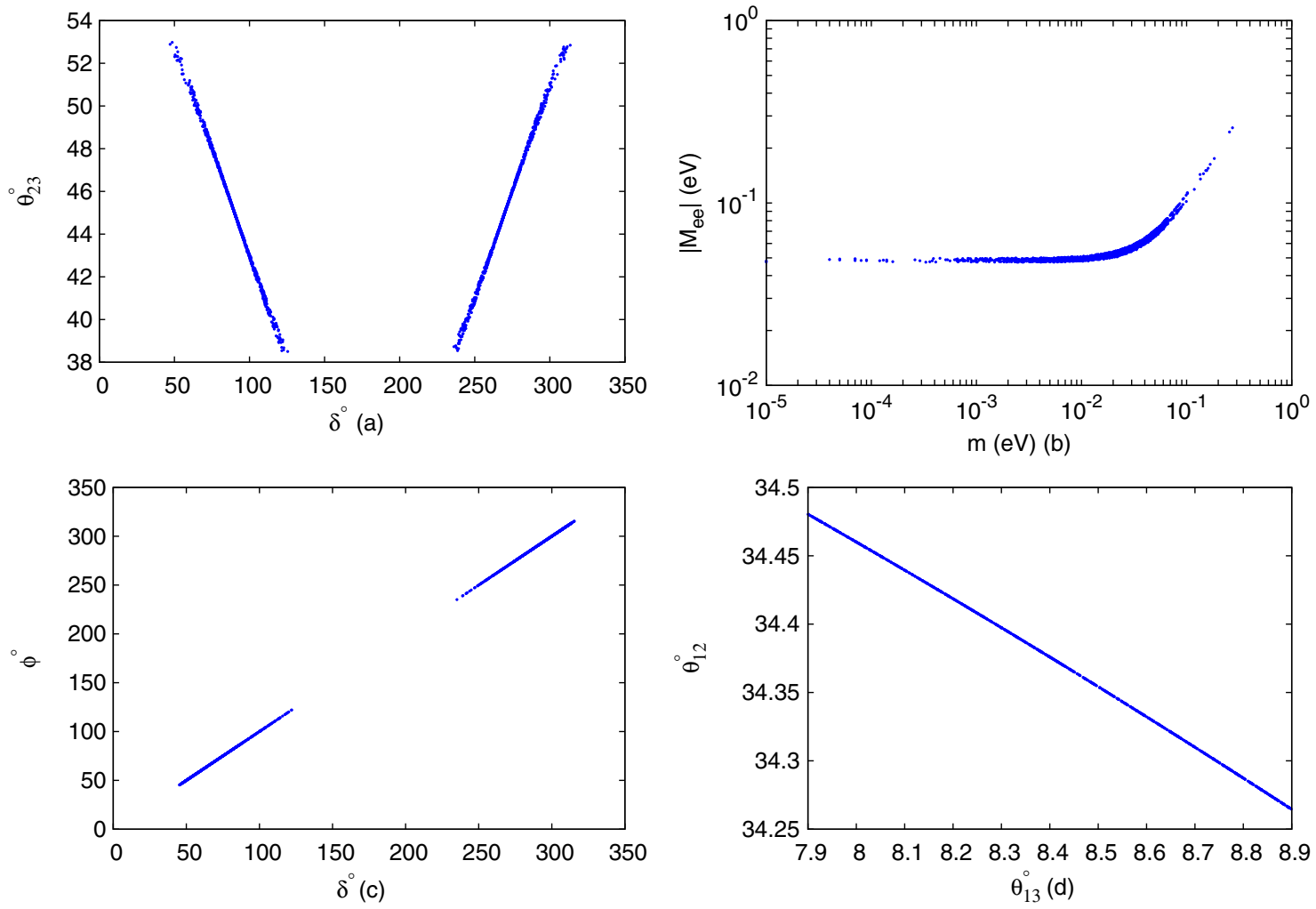

FIG. 5. Correlation plots for patterns $M_{\mathrm{TM}_{1}}^{\mathrm{II}}$ (a),(b), $M_{\mathrm{TM}_{1}}^{\mathrm{IV}}$ (c), and $M_{\mathrm{TM}_{1}}^{\mathrm{V}}$ (d) with IO. 
phase $\delta$ is restricted to two regions around $90^{\circ}$ and $270^{\circ}$. This result holds for all the allowed patterns and is independent of the mass ordering. In the numerical analysis, we have varied the Dirac phase $\delta$ within its full possible range of $0^{\circ}-360^{\circ}$. Recent long baseline neutrino oscillation experiments such as MINOS and T2K [29] have shown a preference for the $C P$ violating phase $\delta$ to be around $270^{\circ}$. Particularly, recent global analysis in Ref. [24] rules out $\delta$ from $32^{\circ}$ to $141^{\circ}$ at $3 \sigma \mathrm{CL}$ for inverted mass ordering. If we take into account, the limits on $\delta$ as given in Ref. [24], the region of $\delta$ around $90^{\circ}$ is ruled out and only the second region around $270^{\circ}$ remains compatible with IO. Figure 4(c) shows the correlation plot between $\delta$ and $J_{C P}$ for pattern $M_{\mathrm{TM}_{1}}^{\mathrm{IV}}$ with NO. It is clear that a vanishing $J_{C P}$ is not allowed for this pattern; in fact, all the patterns with one texture zero and $\mathrm{TM}_{1}$ mixing predict a nonzero $J_{C P}$ which implies that these patterns are necessarily $C P$ violating. This is because these patterns do not allow values $0^{\circ}$ and $180^{\circ}$ for the Dirac phase $\delta$, and since all the mixing angles are nonzero, the $C P$ invariant $J_{C P}$ cannot vanish.

Phases $\phi$ and $\delta$ are found to have almost identical values [Fig. 5(c)] which is similar to the $\mathrm{TM}_{2}$ case. The correlation between mixing angles $\theta_{13}-\theta_{12}$ is shown in Fig. 5(d). In contrast to the $\mathrm{TM}_{2}$ case, here, the value of $\theta_{12}$ decreases with increasing $\theta$. This is a generic feature of $\mathrm{TM}_{1}$ mixing arising from Eq. (55). This brings $\theta_{12}$ near its best-fit experimental value. Thus $\mathrm{TM}_{1}$ mixing is phenomenologically more appealing than $\mathrm{TM}_{2}$ mixing.

\section{SUMMARY}

We studied the implications of having one texture zero in the neutrino mass matrix along with $\mathrm{TM}_{1} / \mathrm{TM}_{2}$ mixing. Considering neutrinos to be Majorana fermions, there are six possible patterns of one texture zero in the neutrino mass matrix. All six patterns are found to be phenomenologically allowed when combined with $\mathrm{TM}_{1} / \mathrm{TM}_{2}$ mixing. The presence of a texture zero in the neutrino mass matrix leads to relations between neutrino masses and mixing matrix elements, whereas $\mathrm{TM}_{1} / \mathrm{TM}_{2}$ mixing implies relations between mixing angles. Thus, the combination of one texture zero patterns with $\mathrm{TM}_{1} / \mathrm{TM}_{2}$ mixing leads to very predictive neutrino mass matrices. For the pattern where the texture zero is at $(1,1)$ position in the neutrino mass matrix, only normal mass ordering is experimentally allowed. Since $\mathrm{TM}_{2}$ mixing predicts values of $\theta_{12}$ away from its best-fit value, $\mathrm{TM}_{1}$ mixing is phenomenologically more appealing. We have obtained predictions for the unknown parameters such as the effective Majorana neutrino mass, the Dirac $C P$ violating phase and the neutrino mass scale. The Dirac phase $\delta$ has been found to be strongly correlated with the phase parameter $\phi$ for both $\mathrm{TM}_{1}$ as well as $\mathrm{TM}_{2}$ mixing. We have also constructed neutrino mass models which lead to patterns of one texture zero with $\mathrm{TM}_{2}$ mixing. To realize these patterns we have employed the $A_{4}$ symmetry within the framework of type-I+II seesaw mechanism.

\section{ACKNOWLEDGMENTS}

R. R. G. acknowledges the financial support provided by the Council of Scientific and Industrial Research (CSIR), Government of India, Grant No. 13(8949-A)/2017-Pool. Part of this work was supported by the Department of Science and Technology, Government of India, Grant No. SB/FTP/PS-128/2013. I thank Sanjeev Kumar and Desh Raj for carefully reading the manuscript.

\section{APPENDIX: GROUP $\boldsymbol{A}_{4}$}

$A_{4}$ has four inequivalent irreducible representations (IRs) which are three singlets $\mathbf{1}, \mathbf{1}^{\prime}$, and $\mathbf{1}^{\prime \prime}$, and one triplet $\mathbf{3}$. The group $A_{4}$ is generated by two generators $S$ and $T$ such that

$$
S^{2}=T^{3}=(S T)^{3}=1 .
$$

The one-dimensional unitary IRs are

$\mathbf{1} S=1 T=1, \quad \mathbf{1}^{\prime} S=1 T=\omega, \quad \mathbf{1}^{\prime \prime} S=1 T=\omega^{2}$.

The three-dimensional unitary IR in the $S$ diagonal basis is

$$
S=\left(\begin{array}{ccc}
1 & 0 & 0 \\
0 & -1 & 0 \\
0 & 0 & -1
\end{array}\right), \quad T=\left(\begin{array}{ccc}
0 & 1 & 0 \\
0 & 0 & 1 \\
1 & 0 & 0
\end{array}\right) .
$$

The multiplication rules of the IRs are as follows:

$\mathbf{1}^{\prime} \otimes 1^{\prime}=1^{\prime \prime}, \quad \mathbf{1}^{\prime \prime} \otimes 1^{\prime \prime}=1^{\prime}, \quad \mathbf{1}^{\prime} \otimes 1^{\prime \prime}=1$.

The product of two 3's gives

$$
\mathbf{3} \otimes \mathbf{3}=\mathbf{1} \oplus \mathbf{1}^{\prime} \oplus \mathbf{1}^{\prime \prime} \oplus \mathbf{3}_{s} \oplus \mathbf{3}_{a}
$$

where $s, a$ denote the symmetric, anti-symmetric products, respectively. Let $\left(x_{1}, x_{2}, x_{3}\right)$ and $\left(y_{1}, y_{2}, y_{3}\right)$ denote the basis vectors of two 3's. IRs obtained from their products are

$$
\begin{gathered}
(\mathbf{3} \otimes \mathbf{3})_{\mathbf{1}}=x_{1} y_{1}+x_{2} y_{2}+x_{3} y_{3} \\
(\mathbf{3} \otimes \mathbf{3})_{\mathbf{1}^{\prime}}=x_{1} y_{1}+\omega^{2} x_{2} y_{2}+\omega x_{3} y_{3} \\
(\mathbf{3} \otimes \mathbf{3})_{\mathbf{1}^{\prime \prime}}=x_{1} y_{1}+\omega x_{2} y_{2}+\omega^{2} x_{3} y_{3} \\
(\mathbf{3} \otimes \mathbf{3})_{\mathbf{3}_{s}}=\left(x_{2} y_{3}+x_{3} y_{2}, x_{3} y_{1}+x_{1} y_{3}, x_{1} y_{2}+x_{2} y_{1}\right) \\
(\mathbf{3} \otimes \mathbf{3})_{\mathbf{3}_{a}}=\left(x_{2} y_{3}-x_{3} y_{2}, x_{3} y_{1}-x_{1} y_{3}, x_{1} y_{2}-x_{2} y_{1}\right) .
\end{gathered}
$$


[1] G. Altarelli and F. Feruglio, Rev. Mod. Phys. 82, 2701 (2010); H. Ishimori, T. Kobayashi, H. Ohki, H. Okada, Y. Shimizu, and M. Tanimoto, Prog. Theor. Phys. Suppl. 183, 1 (2010); S. F. King and C. Luhn, Rep. Prog. Phys. 76, 056201 (2013); S. F. King, J. Phys. G 42, 123001 (2015).

[2] P. F. Harrison, D. H. Perkins, and W. G. Scott, Phys. Lett. B 530, 167 (2002); Z.-Z. Xing, Phys. Lett. B 533, 85 (2002); P. F. Harrison and W. G. Scott, Phys. Lett. B 535, 163 (2002).

[3] K. Abe et al. (T2K Collaboration), Phys. Rev. Lett. 107, 041801 (2011); P. Adamson et al. (MINOS Collaboration), Phys. Rev. Lett. 107, 181802 (2011); Y. Abe et al. (Double Chooz Collaboration), Phys. Rev. Lett. 108, 131801 (2012); F. P. An et al. (Daya Bay Collaboration), Phys. Rev. Lett. 108, 171803 (2012); S.-B. Kim (RENO Collaboration), Phys. Rev. Lett. 108, 191802 (2012).

[4] X. G. He and A. Zee, Phys. Lett. B 645, 427 (2007); Phys. Rev. D 84, 053004 (2011).

[5] C. H. Albright and W. Rodejohann, Eur. Phys. J. C 62, 599 (2009); C. H. Albright, A. Dueck, and W. Rodejohann, Eur. Phys. J. C 70, 1099 (2010).

[6] H. Fritzsch, Z.-z. Xing, and S. Zhou, J. High Energy Phys. 09 (2011) 083; Z.-z. Xing, Phys. Lett. B 530, 159 (2002); B. R. Desai, D. P. Roy, and A. R. Vaucher, Mod. Phys. Lett. A 18, 1355 (2003); A. Merle and W. Rodejohann, Phys. Rev. D 73, 073012 (2006); S. Dev, S. Kumar, S. Verma, and S. Gupta, Nucl. Phys. B784, 103 (2007); S. Dev, S. Kumar, S. Verma, and S. Gupta, Phys. Rev. D 76, 013002 (2007); G. Ahuja, S. Kumar, M. Randhawa, M. Gupta, and S. Dev, Phys. Rev. D 76, 013006 (2007); S. Kumar, Phys. Rev. D 84, 077301 (2011); S. Dev, S. Kumar, and S. Verma, Phys. Rev. D 79, 033011 (2009); P. O. Ludl, S. Morisi, and E. Peinado, Nucl. Phys. B857, 411 (2012); G. Blankenburg and D. Meloni, Nucl. Phys. B867, 749 (2013); W. Grimus and P. O. Ludl, J. Phys. G 40, 055003 (2013); J. Liao, D. Marfatia, and K. Whisnant, J. High Energy Phys. 09 (2014) 013; D. Meloni, A. Meroni, and E. Peinado, Phys. Rev. D 89, 053009 (2014); S. Dev, R. R. Gautam, L. Singh, and M. Gupta, Phys. Rev. D 90, 013021 (2014); G. Ahuja, S. Sharma, P. Fakay, and M. Gupta, Mod. Phys. Lett. A 30, 1530025 (2015); M. Singh, G. Ahuja, and M. Gupta, Prog. Theor. Exp. Phys. 2016, 123B08 (2016); P. H. Frampton, S. L. Glashow, and D. Marfatia, Phys. Lett. B 536, 79 (2002).

[7] L. Lavoura, Phys. Lett. B 609, 317 (2005); E. I. Lashin and N. Chamoun, Phys. Rev. D 78, 073002 (2008); E. I. Lashin and N. Chamoun, Phys. Rev. D 80, 093004 (2009); S. Dev, S. Verma, S. Gupta, and R. R. Gautam, Phys. Rev. D 81, 053010 (2010); S. Dev, S. Gupta, R. R. Gautam, and L. Singh, Phys. Lett. B 706, 168 (2011); T. Araki, J. Heeck, and J. Kubo, J. High Energy Phys. 07 (2012) 083; J. Liao, D. Marfatia, and K. Whisnant, Phys. Rev. D 88, 033011 (2013); J. High Energy Phys. 09 (2014) 013; W. Wang, Phys. Lett. B 733, 320 (2014); 738, 524(E) (2014); Phys. Rev. D 90, 033014 (2014).

[8] S. Kaneko, H. Sawanaka, and M. Tanimoto, J. High Energy Phys. 08 (2005) 073; S. Dev, S. Verma, and S. Gupta, Phys. Lett. B 687, 53 (2010); W. Wang, Eur. Phys. J. C 73, 2551
(2013); S. Dev, R. R. Gautam, and L. Singh, Phys. Rev. D 88, 033008 (2013).

[9] E. I. Lashin and N. Chamoun, Phys. Rev. D 85, 113011 (2012).

[10] R. R. Gautam and S. Kumar, Phys. Rev. D 94, 036004 (2016).

[11] S. Kumar and R. R. Gautam, Phys. Rev. D 96, 015020 (2017).

[12] C. S. Lam, Phys. Rev. D 74, 113004 (2006).

[13] J. D. Bjorken, P. F. Harrison, and W. G. Scott, Phys. Rev. D 74, 073012 (2006).

[14] S. Kumar, Phys. Rev. D 82, 013010 (2010); 88, 016009 (2013).

[15] C. S. Lam, Phys. Lett. B 640 (2006) 260.

[16] C. Jarlskog, Phys. Rev. Lett. 55, 1039 (1985).

[17] I. Abt et al. (GERDA Collaboration), arXiv:hep-ex/0404039.

[18] C. Arnaboldi et al., Nucl. Instrum. Methods Phys. Res., Sect. A 518, 775 (2004).

[19] M. Danilov et al., Phys. Lett. B 480, 12 (2000).

[20] J. J. Gomez-Cadenas et al. (NEXT Collaboration), Adv. High Energy Phys. 2014, 907067 (2014).

[21] R. Gaitskell et al. (Majorana Collaboration), arXiv:nucl-ex/ 0311013.

[22] A. S. Barabash (NEMO Collaboration), Czech. J. Phys. 52, 567 (2002).

[23] P. A. R. Ade et al. (Planck Collaboration), arXiv:1502.01589.

[24] P. F. de Salas, D. V. Forero, C. A. Ternes, M. Tortola, and J. W. F. Valle, arXiv:1708.01186.

[25] P. Minkowski, Phys. Lett. B 67, 421 (1977); T. Yanagida, Proceedings of the Workshop on the Unified Theory and the Baryon Number in the Universe, edited by O. Sawada and A. Sugamoto (KEK, Tsukuba, Japan, 1979), p. 95; M. Gell-Mann, P. Ramond, and R. Slansky, Complex Spinors and Unified Theories in Supergravity, edited by P. Van Nieuwenhuizen and D. Z. Freedman (North Holland, Amsterdam, 1979), p. 315; R. N. Mohapatra and G. Senjanovic, Phys. Rev. Lett. 44, 912 (1980).

[26] W. Konetschny and W. Kummer, Phys. Lett. B 70, 433 (1977); T. P. Cheng and L. F. Li, Phys. Rev. D 22, 2860 (1980); J. Schechter and J. W. F. Valle, Phys. Rev. D 22, 2227 (1980); G. Lazarides, Q. Shafi, and C. Wetterich, Nucl. Phys. B181, 287 (1981); R. N. Mohapatra and G. Senjanovic, Phys. Rev. D 23, 165 (1981).

[27] E. Ma and G. Rajasekaran, Phys. Rev. D 64, 113012 (2001).

[28] S. Gupta, A. S. Joshipura, and K. M. Patel, Phys. Rev. D 85, 031903 (2012); E. Ma, Phys. Rev. D 70, 031901 (2004); E. Ma and D. Wegman, Phys. Rev. Lett. 107, 061803 (2011).

[29] K. Iwamoto, Recent results from T2K and future prospects, in Proceedings of 38th International Conference on High Energy Physics, Chicago, USA, 2016, https://pos.sissa.it/ 282/517; P. Vahle et al. (NOvA Collaborations), New results from NOvA, in Procedings of XXVII International Conference on Neutrino Physics and Astrophysics, London, UK, 2016, http://iopscience.iop.org/article/10.1088/17426596/888/1/012003. 